\section{Research Square}

Preprints are preliminary reports that have not undergone peer review.

They should not be considered conclusive, used to inform clinical practice, or referenced by the media as validated information.

\title{
Medicinal Plants Used in Four Local Government Areas of Southwestern Nigeria for the Management of Diabetes and Its Comorbidities: An Ethnobotanical Survey
}

\section{Latifat O. Sidiq}

Kwara State University

\section{Peter A. Segun}

Olabisi Onabanjo University - Ikenne/Sagamu Campus

Omonike 0 Ogbole ( $\square$ nikeoa@yahoo.com )

University of Ibadan https://orcid.org/0000-0002-6487-9494

\section{Research}

Keywords: Ethnopharmacological survey, Diabetes, Comorbidities, Medicinal plants

Posted Date: May 26th, 2020

DOI: https://doi.org/10.21203/rs.3.rs-30491/v1

License: (c) (i) This work is licensed under a Creative Commons Attribution 4.0 International License. Read Full License 


\section{Abstract}

Background:Diabetes mellitus (DM) continues to pose a major global health threat with serious economic burden in sub-Saharan Africa, especially Nigeria. This condition is often worsened with accompanying comorbidities, further putting a strain on patient wellbeing. Medicinal plants are not only effective in DM, but some of them are also have beneficial effects on accompanying disease states. Such plants may be appropriate alternatives or adjuncts to available antidiabetic medications. This study aimed at accessing and documenting the medicinal plants used in four local government of southwestern Nigeria for the management of diabetes and its comorbidities.

Methods:Semi-structured questionnaires were used to obtain information from traditional medical practitioners, village elders and herb sellers. The data obtained were analyzed and discussed in relation to previously published literature. Fifty-three respondents mostly males (77.4\%) provided information on medicinal plants useful for the management of diabetes and its comorbidities.

Results:A total of 77 medicinal plants belonging to 76 genera in 44 plant families were mentioned in the survey. In particular, 53 medicinal plants are mentioned for managing diabetes, 35 for hypertension, 28 for stroke, 17 for heart diseases and 25 for obesity. The most frequently cited plant family was Leguminosae (12\%), followed by Euphorbiaceae ( $8 \%$ ). The frequently used plant species includes Vernonia amygdalina, Citrus aurantifolia, Viscum album, Carica papaya, Allium sativum. It is interesting to note that only Citrus aurantifoliawas mentioned severally for the management of the five ailments. The herbal recipes, methods of herbal preparation and administration were also recorded.

Conclusions:The survey shows that a significant number ofmedicinal plants are used for the management of diabetes and its comorbidities in the study area. These plants, apart from their hypoglycemic activity, may be useful against the most common complications of DM and may provide strategies for the development of less expensive therapies especially in low income communities.

\section{Introduction}

Medicinal plants have been used for several centuries for the treatment of various ailments and almost seventy thousand species of plant in the world have been screened for their pharmacological activities (1). The importance of the tropical rain forest as a source of raw materials for modern drug development cannot be overemphasized as about $11 \%$ of the 252 drugs considered to be essential drugs are exclusively of plant origin with several others synthesised using natural products template (2). In Nigeria, medicinal plants play prominent role in healthcare delivery as many of her residents, especially those dwelling in the rural areas, rely greatly on medicinal plants and visit traditional medical practitioners (TMPs) for their health care need (3). An earlier study reported that the ratio of TMPs to the population was 1:110, while the ratio of medical doctors to the population was 1:16,400; inferring that TMPs are more accessible to the populace (4). Therefore, herbal medicines continue to play vital role in healthcare delivery, especially in third world nations, where most people have great financial constraints with little or no access to orthodox therapies (3).

Diabetes mellitus (DM), a chronic metabolic disorder associated with abnormally high levels of glucose in the blood, affects approximately $8.3 \%$ of the adult world population and the number of suffers is expected to rise from 382 million in 2013 to 592 million in $2035(5,6)$. There has been an increasing incidence of DM worldwide and this ailment has been shown to pose major health threats and socioeconomic burdens (7). Of the DM sufferers, 22 million reside in sub-Saharan Africa, with Nigeria, the most populous black nation in the world, accounting for a fifth of all diabetes cases in the region (8).

Comorbidity, defined as a situation where one or more chronic ailments occurs in an individual with an index-disease is a common phenomenon amongst DM sufferers (9). In fact, DM has been reported to be a major predisposing factor to the formation of myocardial infarction, cerebrovascular accident and peripheral vascular diseases (10). Common diabetic related comorbidities include obesity, hypertension, chronic kidney diseases, cardiovascular diseases, hyperlipidemia, retinopathy, nephropathy and diabetic foot ulcers $(11,12)$. A recent study conducted in the US between July 2014 and June 2015 revealed that approximately $98 \%$ of DM patients had at least one comorbid condition, while about $89 \%$ had at least two (13).

The debilitating impact of DM comorbidities on patients' health status cannot be underestimated. Comorbidities can drain the financial resources of DM sufferers thereby increasing their out-of-pocket cost for medical care which eventually hinders the ability of the patient to manage their ailments $(14,15)$. In view of this, there is a need to explore alternative means, such as natural sources like medicinal plants, to discover bioactive molecules which may help tackle the menace caused by DM and its related comorbidities. The documentation of indigenous knowledge (IK) has helped in identifying promising medicinal plants; The IK of traditional healers is an asset in the discovery of medicinal plants with therapeutic potentials (16). Several ethnomedical studies on plants used in the management of diabetes have been reported in various parts of the world (1720), In Nigeria, similar researches have been carried out (21-24) regions. However, only few researches have investigated the usage of medicinal plants in the treatment of DM along with its other co-morbidities of. Therefore, this study was carried out to document the various medicinal plants and herbal recipes used in the treatment of DM and its comorbidities and to establish existing relationships between the different recipes for the various diseases with the aim identifying plants that can serve as appropriate alternatives or adjuncts to available antidiabetic medications 


\section{Materials And Methods Study area}

The study was carried out in four local government areas (LGAs) namely: Ibadan (urban; Ibadan North LGA), Ago-Are (rural; Atisbo LGA), IkareAkoko (urban; Akoko North-East LGA) and Oba-Akoko (rural; Akoko South East LGA). The study area (Fig. 1) lies in Oyo and Ondo states of south western Nigeria (Fig. 1). Oyo state lies between latitude $8^{\circ} .12^{\prime} \mathrm{N}$ and longitude $3^{\circ} 42^{\prime} \mathrm{E}$ and covers an area of approximately $28,454 \mathrm{~km}{ }^{2}$ with a population of $110,792(25)$, while Ondo state lies between latitude $6^{\circ} 90^{\prime} \mathrm{N}$ and longitude $4^{\circ} 89^{\prime} \mathrm{E}$ with a land mass of $15,500 \mathrm{~km}^{2}$ and a population of $3,440,000$ as at the 2006 national census (25). The inhabitants of the urban settlers in the study area were mainly educated and involved in high skill jobs, while the rural dwellers were predominately farmers and local traders. The common language spoken by the residents of the study area is Yoruba. The study area has a tropical climate characterised by two distinct weather conditions; rainy season which begins in April and ends in September and the dry season which runs from October to March. The area experiences a relatively high rainfall and humidity for most parts of the year with an average temperature around $32{ }^{\circ} \mathrm{C}$.

\section{Ethnobotanical Survey}

\section{Informed consent}

At the start of the interview, the objective of the study was clearly explained to the intending participants and informed consent to publish the research was sought orally. When the respondents granted consent, the questions contained in the questionnaire were asked and when consent was not given, the individuals were asked no further question.

\section{Plants authentication}

Medicinal plants used for the management of hypertension and its co-morbidities were collected with the aid of the TMP and authenticated by comparison with voucher specimens at the Forest Herbarium, Forestry Research Institute of Nigeria, Ibadan. The names of the plants were verified at the plant database website (www.theplantlist.org; accessed on September 22, 2018).

\section{Data collection}

The study was conducted carried out from May to October, 2014. The ethnomedicinal information of plants used for the management of DM and its comorbidities such as hypertension, obesity, stroke and heart disease were obtained by consulting TMPs, herb sellers and elders in the communities listed above. The use of semi-structured questionnaire via oral interview in the local dialect of the respondents was adopted to obtain the relevant data. Since the interviewer understood the local dialect of the respondents, no interpreter was used in this survey. The questionnaire was divided into three sections. Section 1 deals with demographic information such as age, sex, religion, local tribe, duration of practice, nationality and level of education. Section 2 captures plants used in the treatment of DM and its comorbidities and consisted questions such as frequency of treatment, accompanying side effects and duration of treatment. Section 3 centered on plant parts used, plant availability, local names of diseases, preparation of the recipe and mode of administration of the herbal recipe used in the treatment of diabetes and its comorbidities. Each plant sample was collected at the time of the survey. The botanical names and families of each collected plant samples were authenticated at the Forest Herbarium Ibadan (FHI), Nigeria.

\section{Data analysis}

The Microsoft Office Excel spreadsheet (2016) software was used to conduct descriptive statistical analysis on the socio-demographic data of the respondents, plant part used and plant families. The use-mentions index (UMi) was also calculated and was taken as the frequency of usage of a particular plant for the treatment of diseases divided by the total number of respondents interviewed.

\section{Results And Discussion Demographic data and treatment practice of respondents}

Seventy individuals, including TMPs, herb sellers and village elders, who possess knowledge on the ethnomedicinal use of plants and/or had utilised them for DM and/or its comorbidities in the study area were identified and interviewed. However, only 53 respondents agreed to provide their valuable IK. The demographic information of the respondents is summarized in Table 1 . As can be seen from the table, the majority of the 
respondents were male (77.36\%), a finding similar to other results obtained from several other ethnobotanical studies within the same region (3, $24,26,27)$. This may be unconnected to the cultural perception that the male gender is the heirloom of family heritage making them to easily acquire IK. In addition, it is perceived that the exhaustive and often dangerous practices associated with traditional medicine as exemplified with the collection of medicinal plant from the wild where many wild animals reside may also discourage many female individuals from embracing the practice. Most of the respondents (69.8\%) were Muslims and more than half of the respondents were elderly. Inheritance was the major source of knowledge acquisition as the results presented in Table 1 revealed that $54.7 \%$ of the respondents inherited their knowledge from their parents/ancestors, while only $15.1 \%$ obtained their IK through training. In view of this, it is expedient to document the use of medicinal plant in the treatment of various ailments as this will go a long way in the preservation of IK. Majority of the respondents had the basic (primary) level of education (66\%) with only four of them having no formal education.

Table 1

Demographic data of respondents $(n=53)$

\begin{tabular}{|c|c|c|}
\hline Parameter & Frequency & Percentage (\%) \\
\hline Specialty & 16 & 30.19 \\
\hline Traditional medical practitioner & 14 & 26.42 \\
\hline Herb seller & 23 & 43.39 \\
\hline \multicolumn{3}{|l|}{ Traditional healers } \\
\hline Sex & 41 & 77.36 \\
\hline Male & 12 & 22.64 \\
\hline \multicolumn{3}{|l|}{ Female } \\
\hline Age (years) & 19 & 35.85 \\
\hline $31-50$ & 27 & 50.94 \\
\hline $51-60$ & 7 & 13.21 \\
\hline \multicolumn{3}{|l|}{$>60$} \\
\hline Educational level & 4 & 7.55 \\
\hline None & 30 & 66.04 \\
\hline Primary & 13 & 24.53 \\
\hline Secondary & 1 & 1.89 \\
\hline \multicolumn{3}{|l|}{ Tertiary } \\
\hline Religion & 14 & 26.42 \\
\hline Christianity & 37 & 69.81 \\
\hline Islam & 2 & 3.77 \\
\hline \multicolumn{3}{|l|}{ Traditionalist } \\
\hline Source of knowledge acquisition & 29 & 54.72 \\
\hline Ancestral (Inherited) & 8 & 15.09 \\
\hline Training & 16 & 30.19 \\
\hline Ancestral and Training & & \\
\hline
\end{tabular}

In view of the nature of the practice, the respondents commonly referred to DM as 'itosuga' and claimed they were able to diagnose DM in their patients by examining the presence of sugar in urine which is detected when ants surround the spots of their clients' urine few minutes after urination. Other symptoms that also aided them in the diagnosis of DM included excessive urination, sweating and loss of weight. The respondents referred to hypertension as 'ifunpa giga' and claimed that they normally relied on the laboratory results presented by their patients to diagnose the disease. Stroke, referred to as 'roparoseby the respondents was diagnosed by observing the patient for partial paralysis of a side of the body or the complete paralysis of the body along with impaired speech or vision. For obesity (isanraju), respondents diagnosed the disease by visually observing the fatness of their patients relative to other individuals of the same age and sex.The respondents could not actually differentiate between being overweight and obeseas they claimed the herbal treatments, they prepared were administered to individuals who desired to reduce their body weight, not necessarily obese patients. Heart disease (arunokan) is diagnosed by the respondents when patients have 
symptoms such as persistent irregular heartbeats, shortness of breath and dizziness. The respondents claimed to have successfully treated many patients suffering from these ailments using the various medicinal plant recipes.

\section{Diversity of medicinal plants, herbal preparation and administration}

In the Nigerian ethnomedicine, medicinal plants have been frequently used in the management of human degenerative ailments such as DM and its comorbidities. Medicinal plants have wide patronage due to their accessibility, potency and affordability. There have been several reports of successful treatment of various disease conditions with herbal therapy (28-31). In the present study, 77 medicinal plants belonging to76 genera in 44 plant families were used by the respondents in the management of DM and its co-morbidities. In particular, 53 medicinal plants were used for the management of DM, 35 for hypertension, 28 for stroke, 17 for heart diseases and 25 for obesity (Table 2). The plants mentioned in this survey were readily available as they were easily obtained from the forest, around homes and in the market places. 
Table 2

List of plants used for the management of diabetes and its co-morbidities in the study area

\begin{tabular}{|c|c|c|c|c|c|c|c|c|c|c|c|c|}
\hline \multirow[t]{2}{*}{$\mathrm{S} / \mathrm{N}$} & \multirow[t]{2}{*}{ Family } & \multirow{2}{*}{$\begin{array}{l}\text { Plant Name } \\
\text { (Voucher } \\
\text { specimen no) }\end{array}$} & \multirow[t]{2}{*}{ Common Name } & \multirow{2}{*}{$\begin{array}{l}\text { Local Name } \\
(Y)\end{array}$} & \multirow[t]{2}{*}{ Part Used } & \multicolumn{5}{|c|}{ Therapeutic Use } & \multirow[t]{2}{*}{ UMi } & \\
\hline & & & & & & D & HTN & $S$ & HD & 0 & & \\
\hline 1. & & Acanthaceae & $\begin{array}{l}\text { Acanthus } \\
\text { montanus (Nees) } \\
\text { T. Anderson (FHI } \\
\text { 106492) }\end{array}$ & $\begin{array}{l}\text { Mountain } \\
\text { thistle }\end{array}$ & Ahon-ekun & 1 & & & $x$ & & & 0.019 \\
\hline 2. & & Amaryllidaceae & $\begin{array}{l}\text { Allium cepa L. } \\
\text { (FHI 107561) }\end{array}$ & Onion & Alubosa & bu, & & $x$ & & $x$ & $x$ & 0.075 \\
\hline 3. & & Amaryllidaceae & $\begin{array}{l}\text { Allium sativum L. } \\
\text { (FHI 107576) }\end{array}$ & Garlic & Ayuu & bu & $x$ & $x$ & & $x$ & $x$ & 0.132 \\
\hline 4. & & Anacardiaceae & $\begin{array}{l}\text { Anacardium } \\
\text { occidentale L. } \\
\text { (FHI 109858) }\end{array}$ & Cashew & Kaju & $s$ & $x$ & & & & & 0.038 \\
\hline 5. & & Anacardiaceae & $\begin{array}{l}\text { Mangifera indica } \\
\text { L. (FHI 109451) }\end{array}$ & Mango tree & Mangoro & $\mathrm{I}, \mathrm{s}$ & $x$ & & & & $x$ & 0.038 \\
\hline 6. & & Anacardiaceae & $\begin{array}{l}\text { Spondias } \\
\text { mombin L. (FHI } \\
\text { 106132) }\end{array}$ & Hog plum & lyeye & s & $x$ & & & & & 0.019 \\
\hline 7. & & Annonaceae & $\begin{array}{l}\text { Uvaria afzelii G.F. } \\
\text { Scott-Elliot (FHI } \\
107332)\end{array}$ & Monkey finger & Gbogbonise & $\mathrm{s}$ & & & & & $x$ & 0.019 \\
\hline 8. & & Annonaceae & $\begin{array}{l}\text { Xylopia } \\
\text { aethiopica } \\
\text { (Dunal) A. Rich. } \\
\text { (FHI 10897) }\end{array}$ & $\begin{array}{l}\text { Ethiopian } \\
\text { pepper }\end{array}$ & Eru & $s, f$ & $x$ & $x$ & & $x$ & $x$ & 0.113 \\
\hline 9. & & Apocynaceae & $\begin{array}{l}\text { Alstonia boonei } \\
\text { De Wild. (FHI } \\
\text { 107254) }\end{array}$ & Cheese wood & Ahun & $s, f$ & & & & $x$ & $x$ & 0.038 \\
\hline 10. & & Apocynaceae & $\begin{array}{l}\text { Holarrhena } \\
\text { floribunda (G. } \\
\text { Don) T. Durand \& } \\
\text { Schinz (FHI } \\
\text { 110053) }\end{array}$ & $\begin{array}{l}\text { False rubber } \\
\text { tree }\end{array}$ & Dagba & 1 & & $x$ & & & & 0.019 \\
\hline 11. & & Apocynaceae & $\begin{array}{l}\text { Picralima nitida } \\
\text { (Stapf) T. Durand } \\
\text { \& H. Durand (FHI } \\
\text { 108794) }\end{array}$ & $\begin{array}{l}\text { Akaumma } \\
\text { plant }\end{array}$ & Abere & $s$ & $x$ & $x$ & & $x$ & & 0.019 \\
\hline 12. & & Apocynaceae & $\begin{array}{l}\text { Rauvolfia } \\
\text { vomitoria Afzel. } \\
\text { (FHI 108987) }\end{array}$ & Swizzle stick & Asofeyeje & $s, r$ & $x$ & $x$ & & & $x$ & 0.094 \\
\hline 13. & & Apocynaceae & $\begin{array}{l}\text { Parquetina } \\
\text { nigrescens } \\
\text { (Afzel.) Bullock } \\
\text { (FHI 110044) }\end{array}$ & $\begin{array}{l}\text { African } \\
\text { Parquentina }\end{array}$ & Ogbo & 1 & $x$ & $x$ & & & & 0.075 \\
\hline 14. & & Araceae & $\begin{array}{l}\text { Anchomanes } \\
\text { difformis (Blume) } \\
\text { Engl. (FHI } \\
109585 \text { ) }\end{array}$ & & Aburushoko & $\mathrm{l}, \mathrm{t}$ & $x$ & $x$ & & & & 0.057 \\
\hline 15. & & Bignoniaceae & $\begin{array}{l}\text { Kigelia africana } \\
\text { (Lam.) Benth. } \\
\text { (FHI 107654) }\end{array}$ & Sausage tree & Pandoro & $f$ & $x$ & $x$ & $x$ & & $x$ & 0.094 \\
\hline 16. & & Bignoniaceae & $\begin{array}{l}\text { Newbouldia } \\
\text { laevis (P. Beauv.) } \\
\text { Seem. (FHI } \\
\text { 107753) }\end{array}$ & Fertility tree & Akoko & $\begin{array}{l}\mathrm{I}, \\
\mathrm{s}, \mathrm{r}\end{array}$ & $x$ & & $x$ & & $x$ & 0.094 \\
\hline
\end{tabular}

Key: ae = aerial part; bu = bulb, $f$ = fruits; $I=$ leaves; $r$ ri = rhizome; $r$ = root; $s$ = stem bark; $t$ = tuber

D = Diabetes; HTN = Hypertension; S = Stroke; HD = Hyperlipidemia; O = Obesity 


\begin{tabular}{|c|c|c|c|c|c|c|c|c|c|c|c|c|}
\hline \multirow[t]{2}{*}{$\mathrm{S} / \mathrm{N}$} & \multirow[t]{2}{*}{ Family } & \multirow{2}{*}{$\begin{array}{l}\text { Plant Name } \\
\text { (Voucher } \\
\text { specimen no) }\end{array}$} & \multirow[t]{2}{*}{ Common Name } & \multirow{2}{*}{$\begin{array}{l}\text { Local Name } \\
(Y)\end{array}$} & \multirow[t]{2}{*}{ Part Used } & \multicolumn{5}{|c|}{ Therapeutic Use } & \multirow[t]{2}{*}{ UMi } & \\
\hline & & & & & & D & HTN & s & HD & 0 & & \\
\hline 17. & & Bombacaceae & $\begin{array}{l}\text { Adansonia } \\
\text { digitata L. (FHI } \\
\text { 109806) }\end{array}$ & Baobab & Ose & $f$ & & $x$ & $x$ & & & 0.038 \\
\hline 18. & & Boraginaceae & $\begin{array}{l}\text { Heliotropium } \\
\text { indicum L. (FHI } \\
110156)\end{array}$ & $\begin{array}{l}\text { Indian } \\
\text { heloitrope }\end{array}$ & Apari Igun & ae & $\mathrm{x}$ & $x$ & $x$ & & & 0.057 \\
\hline 19. & & Bromeliaceae & $\begin{array}{l}\text { Ananas comosus } \\
\text { (L.) Merr. (FHI } \\
\text { 107515) }\end{array}$ & Pineapple & Ope oyinbo & $f$ & $\mathrm{x}$ & $x$ & & & & 0.057 \\
\hline 20. & & Caricaceae & $\begin{array}{l}\text { Carica papaya L. } \\
(\mathrm{FHI} 109462)\end{array}$ & Pawpaw & Ibepe & $\mathrm{I}, \mathrm{r}$ & $x$ & $x$ & $x$ & & $x$ & 0.151 \\
\hline 21. & & Combretaceae & $\begin{array}{l}\text { Anogeissus } \\
\text { leiocarpa (DC.) } \\
\text { Guill. \& Perr. (FHI } \\
\text { 107122) }\end{array}$ & Axle wood tree & Ayin & $r$ & & & $x$ & & $x$ & 0.038 \\
\hline 22. & & Compositae & $\begin{array}{l}\text { Acanthospermum } \\
\text { hispidum DC. } \\
\text { (FHI 110050) }\end{array}$ & Goat's head & Dagunro gogoro & ae & $\mathrm{x}$ & $x$ & & & & 0.038 \\
\hline 23. & & Compositae & $\begin{array}{l}\text { Ageratum } \\
\text { conyzoides (L.) L. } \\
\text { (FHI 109634) }\end{array}$ & Goat's weed & Imi-esu & I & $\mathrm{x}$ & $x$ & & & & 0.057 \\
\hline 24. & & Compositae & $\begin{array}{l}\text { Tithonia } \\
\text { diversifolia } \\
\text { (Hemsl.) A. Gray } \\
\text { (FHI 108055) }\end{array}$ & $\begin{array}{l}\text { Mexican } \\
\text { sunflower }\end{array}$ & Sepeleba & I & $\mathrm{x}$ & & $x$ & & & 0.038 \\
\hline 25. & & Compositae & $\begin{array}{l}\text { Vernonia } \\
\text { amygdalina Delile } \\
\text { (FHI 109061) }\end{array}$ & Bitter leaf & Ewuro & I & $x$ & $x$ & $x$ & & $\mathrm{x}$ & 0.208 \\
\hline 26. & & Convolvulaceae & $\begin{array}{l}\text { Ipomoea } \\
\text { asarifolia (Desr.) } \\
\text { Roem. \& Schult. } \\
\text { (FHI 110052) }\end{array}$ & Morning glory & Gboroayaba & I & & $x$ & & & $x$ & 0.057 \\
\hline 27. & & Crassulaceae & $\begin{array}{l}\text { Bryophyllum } \\
\text { pinnatum (Lam.) } \\
\text { Oken (FHI } \\
107762 \text { ) }\end{array}$ & Wonder plant & Odundun & I & $\mathrm{x}$ & $x$ & & & & 0.113 \\
\hline 28. & & Cucurbitaceae & $\begin{array}{l}\text { Lagenaria } \\
\text { breviflora (Benth.) } \\
\text { Roberty (FHI } \\
\text { 109040) }\end{array}$ & Bottle gourd & Tagiri & s & & & & & $x$ & 0.057 \\
\hline 29. & & Cucurbitaceae & $\begin{array}{l}\text { Momordica } \\
\text { charantia L. (FHI } \\
\text { 109638) }\end{array}$ & $\begin{array}{l}\text { African } \\
\text { cucumber }\end{array}$ & Ejinrin & $\mathrm{l}, \mathrm{s}$ & $x$ & & & $x$ & & 0.075 \\
\hline 30. & & Euphorbiaceae & $\begin{array}{l}\text { Alchornea } \\
\text { laxiflora (Benth.) } \\
\text { Pax \& K. Hoffm. } \\
\text { (FHI 110155) }\end{array}$ & $\begin{array}{l}\text { Lowved bead- } \\
\text { string }\end{array}$ & Pepe & I & & $x$ & $x$ & & & 0.038 \\
\hline 31. & & Euphorbiaceae & $\begin{array}{l}\text { Bridelia } \\
\text { ferruginea Benth. } \\
\text { (FHI 109985) }\end{array}$ & Bridelia plant & Irasa & I, s & $x$ & & & & & 0.038 \\
\hline 32. & & Euphorbiaceae & $\begin{array}{l}\text { Croton } \\
\text { gratissimus } \\
\text { Burch. (FHI } \\
\text { 109041) }\end{array}$ & $\begin{array}{l}\text { Lavender } \\
\text { croton }\end{array}$ & Ajeobale & $r$ & & $x$ & & $x$ & & 0.038 \\
\hline 33. & & Euphorbiaceae & $\begin{array}{l}\text { Euphorbia } \\
\text { poissonii Pax } \\
\text { (FHI 109035) }\end{array}$ & Cactus & Oro Adete & s & & & $x$ & & $x$ & 0.057 \\
\hline
\end{tabular}

Key: ae = aerial part; bu = bulb, $\mathrm{f}=$ fruits; $\mathrm{I}=$ leaves; rhi = rhizome; $\mathrm{r}=$ root; $\mathrm{s}=$ stem bark; $\mathrm{t}=$ tuber

D = Diabetes; HTN = Hypertension; S = Stroke; HD = Hyperlipidemia; O = Obesity 


\begin{tabular}{|c|c|c|c|c|c|c|c|c|c|c|c|c|}
\hline \multirow[t]{2}{*}{$\mathrm{S} / \mathrm{N}$} & \multirow[t]{2}{*}{ Family } & \multirow{2}{*}{$\begin{array}{l}\text { Plant Name } \\
\text { (Voucher } \\
\text { specimen no) }\end{array}$} & \multirow[t]{2}{*}{ Common Name } & \multirow{2}{*}{$\begin{array}{l}\text { Local Name } \\
(Y)\end{array}$} & \multirow[t]{2}{*}{ Part Used } & \multicolumn{5}{|c|}{ Therapeutic Use } & \multirow[t]{2}{*}{ UMi } & \\
\hline & & & & & & D & HTN & s & HD & 0 & & \\
\hline 34. & & Euphorbiaceae & $\begin{array}{l}\text { Jatropha curcas } \\
\text { L. (FHl 109020) }\end{array}$ & Nettlespurge & Lapalapa & 1 & $x$ & & $x$ & & & 0.057 \\
\hline 35. & & Euphorbiaceae & $\begin{array}{l}\text { Macaranga } \\
\text { barteri Mülll. Arg. } \\
\text { (FHI 107230) }\end{array}$ & $\begin{array}{l}\text { Macaranga } \\
\text { plant }\end{array}$ & Agbasa & $r$ & $\mathrm{x}$ & & & & & 0.019 \\
\hline 36. & & Gentianaceae & $\begin{array}{l}\text { Anthocleista } \\
\text { djalonensis A. } \\
\text { Chev. (FHI } \\
\text { 109007) }\end{array}$ & Cabbage tree & Sapo & $r, s$ & $\mathrm{x}$ & & & & $x$ & 0.075 \\
\hline 37. & & Hypoxidaceae & $\begin{array}{l}\text { Curculigo pilosa } \\
\text { (Schumach. \& } \\
\text { Thonn.) Engl. } \\
\text { (FHI 109047) }\end{array}$ & $\begin{array}{l}\text { English African } \\
\text { Crocus }\end{array}$ & Epakun & rhi & & & & & $x$ & 0.094 \\
\hline 38. & & Lamiaceae & $\begin{array}{l}\text { Ocimum } \\
\text { gratissimum L. } \\
\text { (FHI 108057) }\end{array}$ & Tea bush & Efinrin & 1 & $\mathrm{x}$ & & $x$ & & & 0.113 \\
\hline 39. & & Lamiaceae & $\begin{array}{l}\text { Solenostemon } \\
\text { monostachyus (P. } \\
\text { Beauv.) Briq. (FHI } \\
\text { 108913) }\end{array}$ & Painted nettle & Olojonguru & ae & & & & $x$ & & 0.038 \\
\hline 40. & & Lauraceae & $\begin{array}{l}\text { Persea americana } \\
\text { Mill. (FHI 109444) }\end{array}$ & Avocado tree & Igba & 1 & & $x$ & $x$ & & & 0.094 \\
\hline 41. & & Leguminosae & $\begin{array}{l}\text { Abrus precatorius } \\
\text { L. (FHI 107452) }\end{array}$ & Cat's eye & Ominsinmisi & 1 & $\mathrm{x}$ & & & $\mathrm{x}$ & & 0.057 \\
\hline 42. & & Leguminosae & $\begin{array}{l}\text { Acacia nilotica } \\
\text { (L.) Delile (FHI } \\
\text { 108425) }\end{array}$ & Prickly acacia & Boonii & s & $\mathrm{x}$ & $x$ & & & & 0.057 \\
\hline 43. & & Leguminosae & $\begin{array}{l}\text { Baphia nitida } \\
\text { Lodd. (FHI } \\
\text { 106544) }\end{array}$ & Camwood & Irosun & I & & $x$ & & & & 0.019 \\
\hline 44. & & Leguminosae & $\begin{array}{l}\text { Cassia fistula L. } \\
\text { (FHI 110261) }\end{array}$ & Purging cassia & Aidantoro & s & & & $x$ & & $x$ & 0.057 \\
\hline 45. & & Leguminosae & $\begin{array}{l}\text { Dialium } \\
\text { guineense Willd. } \\
\text { (FHI 109509) }\end{array}$ & $\begin{array}{l}\text { Velvet } \\
\text { tamarind }\end{array}$ & Irede & $\mathrm{s}, \mathrm{I}$ & & & $x$ & & & 0.038 \\
\hline 46. & & Leguminosae & $\begin{array}{l}\text { Parkia biglobosa } \\
\text { (Jacq.) G. Don } \\
\text { (FHI 107939) }\end{array}$ & $\begin{array}{l}\text { African locust } \\
\text { beans }\end{array}$ & Igi iru & s & $x$ & $x$ & & & & 0.094 \\
\hline 47. & & Leguminosae & $\begin{array}{l}\text { Piliostigma } \\
\text { thonningii } \\
\text { (Schum.) Milne- } \\
\text { Redh. (FHI } \\
\text { 107815) }\end{array}$ & Camel's foot & Abafe & I & & & $x$ & & & 0.019 \\
\hline 48. & & Leguminosae & $\begin{array}{l}\text { Senna alata (L.) } \\
\text { Roxb. (FHI } \\
\text { 108062) }\end{array}$ & Candle bush & Asunwon & 1 & $x$ & & & & & 0.038 \\
\hline 49. & & Leguminosae & $\begin{array}{l}\text { Tetrapleura } \\
\text { tetraptera } \\
\text { (Schum. \& } \\
\text { Thonn.) Taub. } \\
\text { (FHI 110141) }\end{array}$ & Aidan tree & Aidan & $f$ & & $x$ & & $x$ & & 0.095 \\
\hline 50. & & Malvaceae & $\begin{array}{l}\text { Gossypium } \\
\text { barbadense L. } \\
\text { (FHI 107327) }\end{array}$ & Cotton plant & Owu & I, s & $x$ & & & & $x$ & 0.038 \\
\hline
\end{tabular}

Key: $a e=$ aerial part; bu = bulb, $f$ = fruits; $\mathrm{I}=$ leaves; rhi = rhizome; $r$ = root; $s$ = stem bark; $\mathrm{t}=$ tuber D = Diabetes; HTN = Hypertension; S = Stroke; HD = Hyperlipidemia; O = Obesity 


\begin{tabular}{|c|c|c|c|c|c|c|c|c|c|c|c|c|}
\hline \multirow[t]{2}{*}{$S / N$} & \multirow[t]{2}{*}{ Family } & \multirow{2}{*}{$\begin{array}{l}\text { Plant Name } \\
\text { (Voucher } \\
\text { specimen no) }\end{array}$} & \multirow[t]{2}{*}{ Common Name } & \multirow{2}{*}{$\begin{array}{l}\text { Local Name } \\
(Y)\end{array}$} & \multirow[t]{2}{*}{ Part Used } & \multicolumn{5}{|c|}{ Therapeutic Use } & \multirow[t]{2}{*}{ UMi } & \\
\hline & & & & & & D & HTN & $s$ & HD & 0 & & \\
\hline 51. & & Malvaceae & $\begin{array}{l}\text { Hibiscus } \\
\text { sabdariffa L. (FHI } \\
\text { 107622) }\end{array}$ & Roselle & Isapa & $f$ & $\mathrm{x}$ & & & & $x$ & 0.075 \\
\hline 52. & & Meliaceae & $\begin{array}{l}\text { Azadirachta } \\
\text { indica L. (FHI } \\
\text { 109461) }\end{array}$ & Neem tree & Dongoyaro & I & $\mathrm{x}$ & & & & & 0.038 \\
\hline 53. & & Mimosaseae & $\begin{array}{l}\text { Mimosa pudica L. } \\
\text { (FHI 100332) }\end{array}$ & Sensitive plant & Patanmo & I & $\mathrm{x}$ & & & & & 0.038 \\
\hline 54. & & Moraceae & $\begin{array}{l}\text { Artocarpus altilis } \\
\text { (Parkinson ex F. } \\
\text { A. Zorn) Fosberg } \\
\text { (FHI 110483) }\end{array}$ & Breadfruit & $\begin{array}{l}\text { Gberebuutu/Epa } \\
\text { Oyinbo }\end{array}$ & I & $x$ & & $x$ & & & 0.038 \\
\hline 55. & & Moraceae & $\begin{array}{l}\text { Ficus exasperata } \\
\text { Vahl (FHI } \\
109453)\end{array}$ & Fig tree & Epin & 1 & & $x$ & & $x$ & & 0.057 \\
\hline 56. & & Musaceae & $\begin{array}{l}\text { Musa paradisiaca } \\
\text { L. (FHI 110122) }\end{array}$ & Banana & Ogede agbagba & $f$ & $\mathrm{x}$ & & $x$ & & & 0.095 \\
\hline 57. & & Myrtaceae & $\begin{array}{l}\text { Psidium guajava } \\
\text { L. (FHI 109454) }\end{array}$ & Guava tree & Gurofa & s, I & $\mathrm{x}$ & $x$ & & & & \\
\hline 58. & & Olacaceae & $\begin{array}{l}\text { Olax } \\
\text { subscorpioides } \\
\text { Oliv. (FHI 109065) }\end{array}$ & $\begin{array}{l}\text { Stink ant } \\
\text { forest }\end{array}$ & Ifon & I, s & $\mathrm{x}$ & & & & $x$ & 0.057 \\
\hline 59. & & Orchidaceae & $\begin{array}{l}\text { Calyptrochilum } \\
\text { christyanum } \\
\text { (Rchb. f.) } \\
\text { Summerh. (FHI } \\
\text { 110054) }\end{array}$ & $\begin{array}{l}\text { Calyptrochilum } \\
\text { plant }\end{array}$ & Ela & 1 & $\mathrm{x}$ & & & & & 0.038 \\
\hline 60. & & Phyllanthaceae & $\begin{array}{l}\text { Phyllanthus } \\
\text { amarus } \\
\text { Schumach. \& } \\
\text { Thonn. (FHI } \\
\text { 109059) }\end{array}$ & Black catnip & Eyin olobe & $\mathrm{l}, \mathrm{t}$ & $x$ & $x$ & & & & 0.057 \\
\hline 61. & & Piperaceae & $\begin{array}{l}\text { Piper guineense } \\
\text { Schumach. \& } \\
\text { Thonn. (FHI } \\
\text { 110051) }\end{array}$ & $\begin{array}{l}\text { West African } \\
\text { pepper }\end{array}$ & lyere & $f$ & & & $x$ & $x$ & & 0.057 \\
\hline 62. & & Poaceae & $\begin{array}{l}\text { Bambusa } \\
\text { vulgaris Schrad. } \\
\text { (FHI 109052) }\end{array}$ & Bamboo & Oparun & I & $x$ & & $x$ & & & 0.038 \\
\hline 63. & & Poaceae & $\begin{array}{l}\text { Zea mays L. (FHI } \\
\text { 109082) }\end{array}$ & Maize & Agbado & I & & & & $x$ & & 0.038 \\
\hline 64. & & Polygalaceae & $\begin{array}{l}\text { Securidaca } \\
\text { longipedunculata } \\
\text { Fresen. (FHI } \\
\text { 109972) }\end{array}$ & Violet tree & Ipeta & $s, r$ & $\mathrm{x}$ & $x$ & & & & 0.057 \\
\hline 65. & & Rubiaceae & $\begin{array}{l}\text { Morinda lucida } \\
\text { Benth. (FHI } \\
\text { 106992) }\end{array}$ & $\begin{array}{l}\text { Brime stone } \\
\text { tree }\end{array}$ & Oruwo & I & $x$ & & & & & 0.075 \\
\hline 66. & & Rubiaceae & $\begin{array}{l}\text { Nauclea } \\
\text { diderrichii (De } \\
\text { Wild.) Merr. (FHI } \\
\text { 110049) }\end{array}$ & Leichhardt tree & Egbesi & s & $x$ & & $x$ & & & 0.113 \\
\hline 67. & & Rutaceae & $\begin{array}{l}\text { Citrus aurantifolia } \\
\text { (Christm.) } \\
\text { Swingle (FHI } \\
\text { 110009) }\end{array}$ & Lime & Osanwewe & $f$ & $x$ & $x$ & $x$ & $x$ & $x$ & 0.170 \\
\hline
\end{tabular}

Key: $a e=$ aerial part; bu = bulb, $f$ = fruits; $\mathrm{I}=$ leaves; rhi = rhizome; $r$ = root; $s$ = stem bark; $\mathrm{t}=$ tuber

D = Diabetes; HTN = Hypertension; S = Stroke; HD = Hyperlipidemia; O = Obesity 


\begin{tabular}{|c|c|c|c|c|c|c|c|c|c|c|c|c|}
\hline \multirow[t]{2}{*}{$\mathrm{S} / \mathrm{N}$} & \multirow[t]{2}{*}{ Family } & \multirow{2}{*}{$\begin{array}{l}\text { Plant Name } \\
\text { (Voucher } \\
\text { specimen no) }\end{array}$} & \multirow[t]{2}{*}{ Common Name } & \multirow{2}{*}{$\begin{array}{l}\text { Local Name } \\
(Y)\end{array}$} & \multirow[t]{2}{*}{ Part Used } & \multicolumn{5}{|c|}{ Therapeutic Use } & \multirow[t]{2}{*}{ UMi } & \\
\hline & & & & & & D & HTN & s & $H D$ & 0 & & \\
\hline 68. & & Santalaceae & $\begin{array}{l}\text { Viscum album L. } \\
\text { (FHI 108411) }\end{array}$ & Mistletoe & Afomo & 1 & $x$ & $\mathrm{x}$ & & & $\mathrm{x}$ & 0.151 \\
\hline 69. & & Sapindaceae & $\begin{array}{l}\text { Lecaniodiscus } \\
\text { cupanioides } \\
\text { Planch. ex Benth. } \\
\text { (FHI 110081) }\end{array}$ & Lecaniodiscus & Akika & s & $x$ & & $x$ & & & 0.038 \\
\hline 70. & & Sapotaceae & $\begin{array}{l}\text { Vitellaria } \\
\text { paradoxa C. F. } \\
\text { Gaertn. (FHI } \\
\text { 107924) }\end{array}$ & Shea butter & Ori & s & & & & $\mathrm{x}$ & $x$ & 0.057 \\
\hline 71. & & Solanaceae & $\begin{array}{l}\text { Datura metel L. } \\
\text { (FHI 106922) }\end{array}$ & Thorn apple & Gegemu & I & $x$ & & $x$ & & & 0.075 \\
\hline 72. & & Solanaceae & $\begin{array}{l}\text { Solanum } \\
\text { erianthum D. Don } \\
\text { (FHI 106923) }\end{array}$ & Potato tree & Asimau & $\mathrm{I}, \mathrm{r}$ & $x$ & & $x$ & $\mathrm{x}$ & & 0.095 \\
\hline 73. & & Talinaceae & $\begin{array}{l}\text { Talinum } \\
\text { triangular (Jacq.) } \\
\text { Willd. (FHI } \\
\text { 109932) }\end{array}$ & Water leaf & Egbure & ae & $x$ & $\mathrm{x}$ & & & & 0.057 \\
\hline 74. & & Ulmaceae & $\begin{array}{l}\text { Trema orientalis } \\
\text { (L.) Blume (FHI } \\
107813)\end{array}$ & Pigeon wood & Afefe & I, s & $x$ & & $x$ & & & 0.057 \\
\hline 75. & & Xanthorrhoeaceae & $\begin{array}{l}\text { Aloe vera (L.) } \\
\text { Burm. f. (FHI } \\
108024)\end{array}$ & Aloe & Ahon-erin & 1 & $x$ & $\mathrm{x}$ & & & & 0.113 \\
\hline 76. & & Zingiberaceae & $\begin{array}{l}\text { Aframomum } \\
\text { melegueta K. } \\
\text { Schum. (FHI } \\
\text { 109986) }\end{array}$ & $\begin{array}{l}\text { Alligator } \\
\text { pepper }\end{array}$ & Atare & $f$ & $x$ & & $x$ & & & 0.075 \\
\hline 77. & & Zingiberaceae & $\begin{array}{l}\text { Zingiber officinale } \\
\text { Roscoe (FHI } \\
\text { 107935) }\end{array}$ & Ginger & Atale & rhi & $x$ & $x$ & & $\mathrm{x}$ & $\mathrm{x}$ & 0.113 \\
\hline
\end{tabular}

Key: $a e=$ aerial part; $b u=$ bulb, $f$ = fruits; $I$ = leaves; rhi = rhizome; $r$ = root; $s$ = stem bark; $\mathrm{t}=$ tuber

D = Diabetes; HTN = Hypertension; S = Stroke; HD = Hyperlipidemia; O = Obesity

Of the plant species encountered in the study for the treatment of various ailments, trees constituted $45 \%$ of the plant population, while shrubs, herbs and climbers contributed $33 \%, 18 \%$ and $4 \%$, respectively. The analysis of the plant parts used in this study revealed that the predominant plant part was dominated by leaves (43\%), followed by stem (27\%) and fruit (10\%) (Figure 2). Similar occurrences were recorded in previous ethnobotanical studies in several parts of Nigeria $(3,24,32-34)$. This could be connected to the relative availability of leaves for most part of the year and its collection posing the least threat to biodiversity conservation. The most frequently cited plant family was Leguminosae (12\%), followed by Euphorbiaceae (8\%), Apocynaceae (6\%), Compositae (5\%) and Anacardiaceae (4\%), with the remaining plant families having percentage occurrence less than $3 \%$ (Table 2$)$.

The recipes obtained from the respondents were found to be poly-herbals as they believe it is a more holistic method of managing the illness and they claimed it is more potent than the use of individual plant species. Previous studies have revealed that the combination of several plant species in traditional medicine offers synergistic approach to the management of the ailment as each plant will contribute different quota to the therapeutic efficacy of the recipes $(35,36)$. In this study, twenty-three, nineteen, eleven, ten and seven herbal remedies were mentioned for the treatment of diabetes, hypertension, stroke, heart diseases and obesity, respectively (Supplementary material). Various methods employed for the preparation of the herbal recipes were encountered in this study including decoction (43\%), juice (22\%), infusion (20\%), powder ( $9 \%$ ) and paste (6\%). The powdered preparations were used when mixed with hot pap, while decoctions and infusions were usually made with water or pap water. The pap water and hot pap were obtained by soaking dried corn in water for three to four days to soften it after which it was ground to form a smooth paste. To get rid of corn shaft that may be contained in the paste, it is sieved and made to stand for three days. The supernatant is called pap water, whereas hot pap was obtained by boiling the sediment in hot water. The liquid preparations were administered using a cup or table spoon, whereas the pastes were applied externally on the affected part. 
Data obtained from this study revealed that Vernonia amygdalinaDelile was the most frequently used plant with a user mention index (UMi) of 0.208. Other commonly used medicinal plants included Citrus aurantifolia(Christm.) Swingle (UMi 0.170), Viscum album L. (UMi 0.151), Carica papaya L. (UMi 0.151), Allium sativum L. (UMi 0.132), Bryophyllumpinnatum(Lam.) Oken. (UMi 0.113), Xylopia aethiopica (Dunal) A. Rich. (UMi 0.113), Ocimumgratissimum L. (UMi 0.113), Naucleadiderrichii(De Wild.) Merr. (UMi 0.113), Aloe vera(L.) Burm. f. (UMi 0.113) and Zingiber officinaleRoscoe (UMi 0.113). Amongst the plant species, Citrus aurantifoliawas the only plant mentioned by several respondents for the management of the five ailments. Allium sativum,Xylopia aethiopica and Zingiber officinalewere used for the treatment of all the ailments with the exception of stroke, while Carica papaya, Vernonia amygdalina and Kigeliaafricana(Lam.) Benth were used for the management of DM, hypertension, stroke and obesity (Table 2). With the exception of Anacardium occidentaleL, Spondiasmombin L., Brideliaferruginea Benth, Macaranga barterMüll. Arg., Senna alata(L.) Roxb., Calyptrochilumchristyanum (Rchb. f.) and Morinda lucida Benth., all the plant species that were used for the management of DM were also used in the treatment of one or more of its co-morbidities. This finding may suggest that those recipes used for the management of diabetes can as well manage any of its co-morbidities if administered to individuals suffering from any of the ailment. This can also provide relief from the adverse effects of polypharmacy associated with the use of synthetic drugs that is often experienced by sufferers of DM with accompanying comorbidities.

\section{Brief literature review of some of the identified plants used for the management of diabetes and its comorbidities}

Many of the plant species encountered in this research have been investigated for their use in the management of DM and related medical ailments and this may provide support for their use in the study area. A brief description of the antidiabetic potentials and usage in the management of other cardiovascular diseases of some of the frequently mentioned plants is provided below.

Aframomummelegueta(family: Zingiberaceae), commonly referred to as Alligator pepper or grain of paradise, is a tropical plant widely cultivated in many African nations for its edible spicy fruits (37). 6-gingerol and oleanolic acid isolated from the fruits of $A$. melegueta showed strong inhibition of the activity of a-amylase and a-glucosidase, when compared to the standard antidiabetic drug, acarbose, and the antidiabetic effect of the fruits of $A$. meleguetacould be attributed to these compounds (38). The administration of the seeds of $A$. meleguetaproduced significant reduction in several cardiovascular parameters including the systolic blood pressure and mean arterial pressure (MAP) in normotensive and hypertensive human population (39). Adefegha et al reported that the inclusion of $A$. meleguetaseeds in the diet of hypercholesterolemic rats for 30 days significantly reduced the MAP, attenuated hepatotoxicity and elicited antihypercholesterolemic effect suggesting the plant may have therapeutic usefulness in the management of hypertension and obesity (40). In a similar research, the seeds of $A$. meleguetaameliorated the increment in serum triacylglycerol, low-density lipoprotein (LDL) cholesterol and total cholesterol as well as the decrease in the activities of superoxide dismutase, glutathione peroxidase and glutathione reductase caused by the administration of Triton X-100 in hyperlipidemic rats (41).

Allium sativum (garlic) is an important functional food and dietary supplement in many parts of the world.. In particular, there is a wide assumption that they confer protective effect on the heart and prevent several cardiovascular diseases and this has been validated scientifically in several literature (42-45). Garlic significantly reduced the blood glucose levels of STZ-induced diabetic rats as well as attenuated the progression of diabetic structural nephropathy that was observed in non-treated diabetic rats (43), while another similar research reported that the extract markedly reduces the serum levels of triglycerides, creatinine, total cholesterol and LDL-cholesterol (46).

Bryophyllumpinnatum(family: Crassulaceae), commonly referred to as resurrection plant, is a herbaceous plant native to Madagascar but with widespread use in many parts of the world. Several researches have shown that the chronic administration of the aqueous extract of $B$. pinnatum produced hypoglycemic effect in STZ-induced diabetic rats as well as elicited a significant reduction in the serum cholesterol and triglyceride levels (47-49). In a recent study, B. pinnatumreduced lipid peroxidation in heart and liver and exerted antihypercholesterolemia in rabbits(50).

Decoctions and infusions obtained from the morphological parts of Carica papayahave been used traditionally in the management of several ailments including cancer, DTN, diarrhea, inflammation and infectious diseases (51). In an early human study, there was an increase in the serum insulin levels of type 2 diabetic patients that took $602 \mathrm{~g}$ of $C$. papaya fruit; a nutritional dose that is equivalent to $50 \mathrm{~g}$ of carbohydrate (52). The administration of the methanol extract of $C$. papaya leaves to Wistar rats at a twice daily dose of $100 \mathrm{mg} / \mathrm{kg}$ bw displayed remarkable antihypertensive effect as it reduced the plasma angiotensin converting enzyme (ACE) activity and cardiac hypertrophy at levels similar to the positive control, enalapril (53). Other studies showed that the root extract of the plant reduces the MAP of rats in a renovascular model of hypertension (54).

Citrus aurantifolia is a small tree used in African traditional medicine for the management of anorexia, obesity, malaria, DTN and cancer and cough $(3,55)$. Administration of the $C$. aurantifoliajuice in rats with high cholesterol diet intake significantly reduced the levels of LDL and total cholesterol, while causing an increment in the plasma HDL cholesterol levels. In the same study, the juice of the plant inhibited the activity of ACE in a dose dependent manner and also exerted potent antioxidant potential in various in vitro antioxidant assays including DPPH and nitric oxide scavenging assays (56). In a 45-day study, the simultaneous administration of the essential oils of $C$. aurantifoliawith ketotifen significantly reduced the consumption of food and caused drastic weight loss in experimental mice (57). 
Persea americana, a tree of about $20 \mathrm{~m}$ and indigenous to South Central Mexico, is used in traditional medicine for the treatment of hypertension (58), DTN (59), renal diseases (60) and obesity (61). Phenolic compounds including protocatechuic acid, kaempferide and vanillic acid present in the methanol extract of the seeds of $P$. americana were thought to be responsible for the significant reduction in the LDL and total cholesterol levels of mice on high hyperlipidemic diet (62). An earlier study revealed that methanol extract of the leaves of $P$. americana induced a transient reduction in MAP in anaesthetized normotensive rats (63), while another in vivo study demonstrated that the extract displayed significant vasorelaxant effect on isolated thoracic rat aorta (64). The chronic administration (28 days) of the hydroethanolic extract of $P$. americana in STZinduced diabetic rats decreased the blood glucose levels and enhanced the metabolic activity of the experimental animals; an effect probably due to the activation of the protein kinase B enzymatic pathway (65).

Vernonia amygdalina (Asteraceae), a shrub indigenous to tropical Africa, is probably the most widely used member of the Vernonia genus for medicinal purpose. Several researches have documented the use of $V$. amygdalinain the management of DTN and vascular diseases. The aqueous extract $(80 \mathrm{mg} / \mathrm{kg}$ ) of the leaves of $V$. amygdalinawas shown to induce a dose-dependent blood sugar lowering effects in alloxan-induced diabetic rabbits (66), while the chronic administration of its ethanolic extract (400 mg/kg) was reported to elicit a hypoglycemic effect as well as induce the expression of Glut 4 receptors in STZ-induced diabetic rats. In a study demonstrating the lipid lowering effects of the methanolic extract of $V$. amygdalinaconducted in rats fed with high cholesterol diet, $V$. amygdalinaat a dose of $200 \mathrm{mg} / \mathrm{kg}$ induced $49 \%$ dose-dependent decrease in LDL cholesterol, while it increased the levels of HDL by $59 \%$. In addition, the extract significantly decreased the levels of plasma and post mitochondrial fraction lipid peroxidation by $42 \%$ and $45 \%$, respectively (67).

Viscum album (Loranthaceae), commonly referred to as African mistletoe, is used for the management of abdominal discomfort, anxiety, DTN, epilepsy, hypertension and stroke (68). Nkanuand co-workers reported that the crude extract of $V$. album at a dose of $150 \mathrm{mg} / \mathrm{kg}$ bw significantly reduced the blood glucose and MAP of diabetic and hypertensive rats by about $84.2 \%$ and $34.5 \%$, respectively (69). The methanol extract of $V$. album exerted anti-diabetic and anti-hyperlipidemic effects in STZ-induced diabetic rats by decreasing the fasting blood glucose level by $34 \%$ and significantly reducing the levels of serum triglycerides, lactate dehydrogenase and LDL cholesterol, following a chronic administration (21 days) of the extract at a dose of $100 \mathrm{mg} / \mathrm{kg} \mathrm{bw}(70)$.

Xylopiaaethiopica'sfruits and seeds are used for culinary purposes in many regions of Africa (71). In a recent study, dietary supplementation of hypercholesterolemic rats with Ethiopian pepper fruits significantly reduced the plasma ACE and total cholesterol levels, as well as reversed the elevated serum levels of alanine aminotransferase and aspartate transaminase caused by the high cholesterol diet. Phenolic compounds including rutin, quercetin and ellagic acid were accounted to be responsible for the observed antihypertensive and hypolipidemic properties (72).

Administration of a 50:50 mixture of Alstoniacongensisbark and Xylopia aethiopica fruits had a significant hypoglycemic effect in normoglycemic rats (73), while the chronic administration of a herbal mixture (Okudiabet $\left.{ }^{\circledR}\right)$, which contains $X$. aethiopica as one of its principal constituents, markedly reduced the blood glucose levels in alloxan-induced diabetic rats (74).

Zingiber officinale (ginger) has a long historical use in traditional medicine in various culture and has been scientifically validated for the treatment of several diseases including DTN, hypertension and other cardiovascular ailments (75). The ethanolic extract of ginger significantly lowered the several serum lipid parameters such as total cholesterol, triglycerides and LDL-cholesterol and also protected the pancreas and liver from lipid peroxidation in STZ-induced diabetic rats $(76,77)$. Administration of the ethanolic extract of $Z$. officinalerhizomes produced a dose-dependent reduction in blood glucose levels in normoglycemic and STZ-induced diabetic rats (75), while another study revealed that a single administration of ginger juice attenuated 5-hydroxytryptamine induced hyperglycaemia in diabetic rats (78). Several in vitro studies have reported the inhibitory activity of Z. officinaleon AA-induced platelet aggregation, COX-derived thromboxanes and prostaglandin synthesis (79-81).

\section{Conclusion}

To summarize our findings, we present in this report the inventory of medicinal plants in four local government areas of southwestern Nigeria used for the management of DTN and its comorbidities. A total of 77 medicinal plants belonging to 76 genera in 44 plant families were mentioned by the respondents. In particular, 53 medicinal plants were used for the management of DTN, 35 for hypertension, 28 for stroke, 17 for heart diseases and 25 for obesity. The most frequently cited plant family was Leguminosae (12\%), followed by Euphorbiaceae (8\%), Apocynaceae (6\%), Compositae (5\%) and Anacardiaceae (4\%) with the remaining plant families having percentage occurrence less than $3 \%$.. While only Citrus aurantifoliawas mentioned by several respondents for the management of the five ailments, review of literature has confirmed the multiple effect of some of the key plants on diabetes and it's commorbidities. As most of the respondents encountered in this study belong to the elderly population, this documentation will serve a great deal in preserving the indigenous traditional knowledge of the local communities.Since most diabetic patients often have one or more accompanying comorbidities, a safe and efficacious herbal preparation that can manage both diabetes and its comorbidities may provide scientific breakthrough especially for the local communities.

\section{Declarations}

\section{Ethics approval and consent to participate.}


Informed consent was obtained from each participant according to the guideline of Research Ethics Committee of the University of Ibadan. A copy of the form used is available upon request.

\section{Consent for publication}

Not applicable in this study

\section{Availability of data and materials}

All data generated or analysed during this study are included in this published article (and its supplementary information files).

\section{Competing interests}

The authors declare that they have no competing interests

\section{Funding}

This research did not receive any specific grant from funding agencies in the public, commercial, or not-for-profit sectors.

\section{Authors Contribution}

000 and LOS conceived and designed the work. The interview was conducted by LOS under the supervision and guidance of OOO. Literature search and data analysis were done by LOS and PAS. The first draft of the manuscript was written by LOS and carefully revised by PAS. All authors agreed on the final draft of the manuscript.

\section{Acknowledgments}

We gratefully acknowledge the respondents for their cooperation and willingness to share their knowledge on medicinal plants used for the management of various ailments. The authors are greatly indebted to the staff of the Forestry Research Institute of Nigeria (FRIN) for the authentication of the plant sample.

\section{Abbreviations}

ACE, angiotensin-I converting enzyme; ADL, adenosine diphosphate; BW, body weight; DM, diabetes mellitus; FHI, forest herbarium Ibadan; HDL, high-density lipoprotein; IK, indigenous knowledge; LDL, low-density lipoprotein; LGAs, local government areas; MAP, mean arterial pressure; STZ, streptozotocin; TMPs, traditional medical practitioners; UMi, use-mentions index; WHO, world health organization

\section{References}

1. Zhang Q. Global situation and WHO strategy on traditional medicine. Traditional Medicine and Modern Medicine. 2018;1(01):11-3.

2. Cragg G, Newman D. Natural products and drug discovery and development: A history of success and continuing promise for the future. Planta Medica. 2014;80(10):IL1.

3. Segun PA, Ogbole OO, Ajaiyeoba EO. Medicinal Plants Used in the Management of Cancer among the ljebus of Southwestern Nigeria. Journal of Herbal Medicine. 2018.

4. Oladele A, Adewunmi C. Medicinal Plants Used In The Management Of Malaria Among The Traditional Medicine Practitioners (TMP I's) In South Western Nigeria. African Journal of Infectious Diseases. 2008;2(1):51-9.

5. Shaw JE, Sicree RA, Zimmet PZ. Global estimates of the prevalence of diabetes for 2010 and 2030. Diabetes Research and Clinical Practice. 2010;87:4-14.

6. Rahimi M. A review: anti diabetic medicinal plants used for diabetes mellitus. Bull Env Pharmacol Life Sciences. 2015;4:163-80.

7. Dahiru T, Aliyu AA, Shehu A. A review of population-based studies on diabetes mellitus in Nigeria. Sub-Saharan African Journal of Medicine. 2016;3(2):59.

8. Fasanmade OA, Dagogo-Jack S. Diabetes care in Nigeria. Annals of global health. 2015;81(6):821-9. 
9. Struijs JN, Baan CA, Schellevis FG, Westert GP, van den Bos GA. Comorbidity in patients with diabetes mellitus: impact on medical health care utilization. BMC health services research. 2006;6(1):84.

10. Beckman JA, Creager MA, Libby P. Diabetes and atherosclerosis: epidemiology, pathophysiology, and management. Jama. 2002;287(19):2570-81.

11. Kayama Y, Raaz U, Jagger A, Adam M, Schellinger IN, Sakamoto M, et al. Diabetic cardiovascular disease induced by oxidative stress. International journal of molecular sciences. 2015;16(10):25234-63.

12. P Hoffman R. Vascular endothelial dysfunction and nutritional compounds in early type 1 diabetes. Current diabetes reviews. 2014;10(3):2017.

13. Iglay K, Hannachi H, Joseph Howie P, Xu J, Li X, Engel SS, et al. Prevalence and co-prevalence of comorbidities among patients with type 2 diabetes mellitus. Current medical research and opinion. 2016;32(7):1243-52.

14. Noël PH, Chris Frueh B, Larme AC, Pugh JA. Collaborative care needs and preferences of primary care patients with multimorbidity. Health Expectations. 2005;8(1):54-63.

15. Piette JD, Kerr EA. The impact of comorbid chronic conditions on diabetes care. Diabetes care. 2006;29(3):725-31.

16. Ogbole O, Segun P, Fasinu P. Antimicrobial and antiprotozoal activities of twenty-four Nigerian medicinal plant extracts. South African Journal of Botany. 2018;117:240-6.

17. Kadir MF, Sayeed MSB, Shams T, Mia M. Ethnobotanical survey of medicinal plants used by Bangladeshi traditional health practitioners in the management of diabetes mellitus. Journal of ethnopharmacology. 2012;144(3):605-11.

18. Jouad H, Haloui M, Rhiouani H, El Hilaly J, Eddouks M. Ethnobotanical survey of medicinal plants used for the treatment of diabetes, cardiac and renal diseases in the North centre region of Morocco (Fez-Boulemane). Journal of ethnopharmacology. 2001;77(2-3):175-82.

19. Karou SD, Tchacondo T, Djikpo Tchibozo MA, Abdoul-Rahaman S, Anani K, Koudouvo K, et al. Ethnobotanical study of medicinal plants used in the management of diabetes mellitus and hypertension in the Central Region of Togo. Pharmaceutical biology. 2011;49(12):1286-97.

20. Gürdal B, Kültür Ş. An ethnobotanical study of medicinal plants in Marmaris (Muğla, Turkey). Journal of ethnopharmacology. 2013;146(1):113-26.

21. Shinkafi TS, Bello L, Hassan SW, Ali S. An ethnobotanical survey of antidiabetic plants used by Hausa-Fulani tribes in Sokoto, Northwest Nigeria. Journal of ethnopharmacology. 2015;172:91-9.

22. Olatunji B, Ajibola D, Adebayo E, Nyong E, Moody J. Ethnomedicinal survey of botanicals used by herbal practitioners in Yagba East Local Government of Kogi State, Nigeria. European Journal of Medicinal Plants. 2014;4(12):1479.

23. Abo KA, Fred-Jaiyesimi AA, Jaiyesimi AEA. Ethnobotanical studies of medicinal plants used in the management of diabetes mellitus in South Western Nigeria. Journal of ethnopharmacology. 2008;115(1):67-71.

24. Gbolade AA. Inventory of antidiabetic plants in selected districts of Lagos State, Nigeria. Journal of ethnopharmacology. 2009;121(1):135-9.

25. NPC. Population and housing census of the Federal Republic of Nigeria 2006. 2010.

26. Taye OR. Yoruba traditional medicine and the challenge of integration. The Journal of Pan African Studies. 2009;3(3):73-90.

27. Ogbole OO, Gbolade AA, Ajaiyeoba EO. Ethnobotanical survey of plants used in treatment of inflammatory diseases in Ogun State of Nigeria. European Journal of Scientific Research. 2010;43(2):183-91.

28. Gurib-Fakim A. Medicinal plants: traditions of yesterday and drugs of tomorrow. Molecular aspects of Medicine. 2006;27(1):1-93.

29. Ogbole O0, Akinleye TE, Segun PA, Faleye TC, Adeniji AJ. In vitro antiviral activity of twenty-seven medicinal plant extracts from Southwest Nigeria against three serotypes of echoviruses. Virology journal. 2018;15(1):110.

30. Fennell C, Lindsey K, McGaw L, Sparg S, Stafford G, Elgorashi E, et al. Assessing African medicinal plants for efficacy and safety: pharmacological screening and toxicology. Journal of ethnopharmacology. 2004;94(2-3):205-17.

31. Sidiq LO, Segun PA, Ogbole 00. Total Phenolic Contents and Antioxidant Activity of Nine Medicinal Plants used in Nigerian Traditional Medicine. Tropical Journal of Natural Product Research. 2018;2(9):438-41.

32. Sonibare M, Moody J, Adesanya E. Use of medicinal plants for the treatment of measles in Nigeria. Journal of ethnopharmacology. 2009;122(2):268-72.

33. Odoh UE, Uzor PF, Eze CL, Akunne TC, Onyegbulam CM, Osadebe PO. Medicinal plants used by the people of Nsukka Local Government Area, south-eastern Nigeria for the treatment of malaria: An ethnobotanical survey. Journal of ethnopharmacology. 2018;218:1-15.

34. Dike IP, Obembe 00, Adebiyi FE. Ethnobotanical survey for potential anti-malarial plants in south-western Nigeria. Journal of ethnopharmacology. 2012;144(3):618-26.

35. Ebong PE, Atangwho IJ, Eyong EU, Egbung GE. The antidiabetic efficacy of combined extracts from two continental plants: Azadirachta indica (A. Juss)(Neem) and Vernonia amygdalina (Del.)(African bitter leaf). American Journal of Biochemistry and Biotechnology. 2008;4(3):239-44.

36. de Wet H, Nkwanyana MN, van Vuuren SF. Medicinal plants used for the treatment of diarrhoea in northern Maputaland, KwaZulu-Natal Province, South Africa. Journal of ethnopharmacology. 2010;130(2):284-9. 
37. Ilic N, Schmidt BM, Poulev A, Raskin I. Toxicological evaluation of grains of paradise (Aframomum melegueta)[Roscoe] K. Schum. Journal of ethnopharmacology. 2010;127(2):352-6.

38. Mohammed A, Gbonjubola VA, Koorbanally NA, Islam MS. Inhibition of key enzymes linked to type 2 diabetes by compounds isolated from Aframomum melegueta fruit. Pharmaceutical biology. 2017;55(1):1010-6.

39. Lawal B, Aderibigbe A, Essiet G, Essien A. Hypotensive and antihypertensive effects of Aframomum melegueta seeds in humans. Int J Pharmacol. 2007;3:311-8.

40. Adefegha SA, Oboh G, Adefegha OM, Henle T. Alligator pepper/Grain of paradise (Aframomum melegueta) modulates Angiotensin-I converting enzyme activity, lipid profile and oxidative imbalances in a rat model of hypercholesterolemia. Pathophysiology. 2016;23(3):191-202.

41. Adigun N, Oladiji A, Ajiboye T. Antioxidant and anti-hyperlipidemic activity of hydroethanolic seed extract of Aframomum melegueta K. Schum in Triton X-100 induced hyperlipidemic rats. South African Journal of Botany. 2016;105:324-32.

42. Koscielny J, Klüssendorf D, Latza R, Schmitt R, Radtke H, Siegel G, et al. The antiatherosclerotic effect of Allium sativum. Atherosclerosis. 1999;144(1):237-49.

43. Al-Qattan K, Thomson M, Ali M. Garlic (Allium sativum) and ginger (Zingiber officinale) attenuate structural nephropathy progression in streptozotocin-induced diabetic rats. e-SPEN, the European e-Journal of Clinical Nutrition and Metabolism. 2008;3(2):e62-e71.

44. Ali M, Thomson M. Consumption of a garlic clove a day could be beneficial in preventing thrombosis. Prostaglandins, leukotrienes and essential fatty acids. 1995;53(3):211-2.

45. Al-Qattan K, Khan I, Alnaqeeb M, Ali M. Mechanism of garlic (Allium sativum) induced reduction of hypertension in $2 \mathrm{~K}-1 \mathrm{C}$ rats: a possible mediation of $\mathrm{Na} / \mathrm{H}$ exchanger isoform-1. Prostaglandins, Leukotrienes and Essential Fatty Acids. 2003;69(4):217-22.

46. Eidi A, Eidi M, Esmaeili E. Antidiabetic effect of garlic (Allium sativum L.) in normal and streptozotocin-induced diabetic rats. Phytomedicine. 2006;13(9-10):624-9.

47. Ogbonnia SO, Odimegwu Jl, Enwuru VN. Evaluation of hypoglycaemic and hypolipidaemic effects of aqueous ethanolic extracts of Treculia africana Decne and Bryophyllum pinnatum,/i> Lam. and their mixture on streptozotocin (STZ)-induced diabetic rats. African Journal of Biotechnology. 2008;7(15).

48. Ojewole JA. Antinociceptive, anti-inflammatory and antidiabetic effects of Bryophyllum pinnatum (Crassulaceae) leaf aqueous extract. Journal of ethnopharmacology. 2005;99(1):13-9.

49. Menon N, Sparks J, Omoruyi F. Hypoglycemic and hypocholesterolemic activities of the aqueous preparation of Kalanchoe pinnata leaves in streptozotocin-induced diabetic rats. Asian Pacific Journal of Tropical Biomedicine. 2015;5(1):3-9.

50. Adekunle AS, Adelusi TI, Oyewo EB, Kamdem J-P, Akintade BB. Antihypercholesterolemic, Cardioprotective and Vitamins E and C Sparing Properties of Bryophyllum pinnatum in Rabbits. European Journal of Medicinal Plants. 2016;11(3):1-13.

51. Chávez-Quintal P, González-Flores T, Rodríguez-Buenfil I, Gallegos-Tintoré S. Antifungal activity in ethanolic extracts of Carica papaya L. cv. Maradol leaves and seeds. Indian journal of microbiology. 2011;51(1):54-60.

52. Fatema K, Ali L, Rahman MH, Parvin S, Hassan Z. Serum glucose and insulin response to mango and papaya in type 2 diabetic subjects. Nutrition Research. 2003;23(1):9-14.

53. Brasil GA, Ronchi SN, Do Nascimento AM, de Lima EM, Romão W, Da Costa HB, et al. Antihypertensive effect of carica papaya via a reduction in ACE activity and improved baroreflex. Planta Med. 2014;80(17):1580-7.

54. Ravikant T, Nishant G, ShAS Hipal S, Samriti T, Kumar T, Vikas V, et al. Antihypertensive effect of ethanolic extract of Indian Carica papaya L. root bark (Caricaceae) in renal artery occluded hypertensive rats. Int J Pharm Clin Res. 2012;4:20-3.

55. Aibinu I, Adenipekun T, Adelowotan T, Ogunsanya T, Odugbemi T. Evaluation of the antimicrobial properties of different parts of Citrus aurantifolia (lime fruit) as used locally. African Journal of Traditional, Complementary, and Alternative Medicines. 2007;4(2):185.

56. Oboh G, Bello FO, Ademosun AO, Akinyemi AJ, Adewuni TM. Antioxidant, hypolipidemic, and anti-angiotensin-1-converting enzyme properties of lemon (Citrus limon) and lime (Citrus aurantifolia) juices. Comparative Clinical Pathology. 2015;24(6):1395-406.

57. Asnaashari S, Delazar A, Habibi B, Vasfi R, Nahar L, Hamedeyazdan S, et al. Essential Oil from Citrus aurantifolia prevents ketotifen-induced weight-gain in mice. Phytotherapy Research. 2010;24(12):1893-7.

58. Yasir M, Das S, Kharya M. The phytochemical and pharmacological profile of Persea americana Mill. Pharmacognosy reviews. 2010;4(7):77.

59. Andrade-Cetto A, Heinrich M. Mexican plants with hypoglycaemic effect used in the treatment of diabetes. Journal of ethnopharmacology. 2005;99(3):325-48.

60. Agra MdF, Freitas PFd, Barbosa-Filho JM. Synopsis of the plants known as medicinal and poisonous in Northeast of Brazil. Revista Brasileira de Farmacognosia. 2007;17(1):114-40.

61. López RL, Frati AM, Hernández BD, Cervantes SM, Hernández ML, Juárez C, et al. Monounsaturated fatty acid (avocado) rich diet for mild hypercholesterolemia. Archives of medical research. 1996;27(4):519-23.

62. Pahua-Ramos ME, Ortiz-Moreno A, Chamorro-Cevallos G, Hernández-Navarro MD, Garduño-Siciliano L, Necoechea-Mondragón H, et al. Hypolipidemic effect of avocado (Persea americana Mill) seed in a hypercholesterolemic mouse model. Plant foods for human nutrition.

Page $15 / 17$ 
2012;67(1):10-6.

63. Adeboye J, Fajonyomi M, Makinde J, Taiwo O. A preliminary study on the hypotensive activity of Persea americana leaf extracts in anaesthetized normotensive rats. Fitoterapia. 1999;70(1):15-20.

64. Owolabi MA, Jaja SI, Coker HA. Vasorelaxant action of aqueous extract of the leaves of Persea americana on isolated thoracic rat aorta. Fitoterapia. 2005;76(6):567-73.

65. Lima C, Vasconcelos C, Costa-Silva J, Maranhão C, Costa J, Batista T, et al. Anti-diabetic activity of extract from Persea americana Mill. leaf via the activation of protein kinase B (PKB/Akt) in streptozotocin-induced diabetic rats. Journal of ethnopharmacology. 2012;141(1):517-25.

66. Akah P, Okafor C. Blood sugar lowering effect of Vernonia amygdalina Del, in an experimental rabbit model. Phytotherapy Research. 1992;6(3):171-3.

67. Adaramoye OA, Akintayo $\mathrm{O}$, Achem J, Fafunso MA. Lipid-lowering effects of methanolic extract of Vernonia amygdalina leaves in rats fed on high cholesterol diet. Vascular health and risk management. 2008;4(1):235.

68. Hutt N, Kopferschmitt-Kubler M, Cabalion J, Purohit A, Alt M, Pauli G. Anaphylactic reactions after therapeutic injection of mistletoe (Viscum album L.). Allergologia et immunopathologia. 2001;29(5):201-3.

69. Nkanu E, Eno A, Ofem O, Imoru O, Unoh F. Effect of crude extract of Viscum album (mistletoe) on plasma lipids: An insight into its possible antihyperglycaemic and antihypertensive properties. Port Harcourt Journal Medicine. 2007;1(3):171-7.

70. Adaramoye O, Amanlou M, Habibi-Rezaei M, Pasalar P, Ali M-M. Methanolic extract of African mistletoe (Viscum album) improves carbohydrate metabolism and hyperlipidemia in streptozotocin-induced diabetic rats. Asian Pacific journal of tropical medicine. 2012;5(6):427-33.

71. Erhirhie EO, Moke GE. Xylopia Aethiopica: A Review of its Ethnomedicinal, chemical and pharmacological properties. American Journal of Pharmtech Research. 2014;4:22-37.

72. Adefegha SA, Oboh G, Olasehinde TA, Boligon AA. Dietary supplementation with Ethiopian pepper (Xylopia aethiopica) modulates angiotensinI converting enzyme activity, antioxidant status and extenuates hypercholesterolemia in high cholesterol fed Wistar rats. PharmaNutrition. 2018;6(1):9-16.

73. Ogbonnia S, Adekunle A, Bosa M, Enwuru V. Evaluation of acute and subacute toxicity of Alstonia congensis Engler (Apocynaceae) bark and Xylopia aethiopica (Dunal) A. Rich (Annonaceae) fruits mixtures used in the treatment of diabetes. African Journal of Biotechnology. 2008;7(6).

74. Ogbonnia S, Mbaka G, Adekunle A, Anyika E, Gbolade O, Nwakakwa N. Effect of a poly-herbal formulation, Okudiabet, on alloxan-induced diabetic rats. Agriculture and Biology Journal of North America. 2010;1(2):139-45.

75. Ojewole JA. Analgesic, antiinflammatory and hypoglycaemic effects of ethanol extract of Zingiber officinale (Roscoe) rhizomes (Zingiberaceae) in mice and rats. Phytotherapy Research: An International Journal Devoted to Pharmacological and Toxicological Evaluation of Natural Product Derivatives. 2006;20(9):764-72.

76. Bhandari U, Pillai K. Effect of ethanolic extract of Zingiber officinale on dyslipidaemia in diabetic rats. Journal of ethnopharmacology. 2005;97(2):227-30.

77. Al-Amin ZM, Thomson M, Al-Qattan KK, Peltonen-Shalaby R, Ali M. Anti-diabetic and hypolipidaemic properties of ginger (Zingiber officinale) in streptozotocin-induced diabetic rats. British journal of nutrition. 2006;96(4):660-6.

78. Akhani SP, Vishwakarma SL, Goyal RK. Anti-diabetic activity of Zingiber officinale in streptozotocin-induced type I diabetic rats. Journal of pharmacy and Pharmacology. 2004;56(1):101-5.

79. Verma S, Singh M, Jain P, Bordia A. Protective effect of ginger, Zingiber officinale Rosc on experimental atherosclerosis in rabbits. 2004.

80. Koo KL, Ammit AJ, Tran VH, Duke CC, Roufogalis BD. Gingerols and related analogues inhibit arachidonic acid-induced human platelet serotonin release and aggregation. Thrombosis research. 2001;103(5):387-97.

81. Nurtjahja-Tjendraputra E, Ammit AJ, Roufogalis BD, Tran VH, Duke CC. Effective anti-platelet and COX-1 enzyme inhibitors from pungent constituents of ginger. Thrombosis research. 2003;111(4-5):259-65.

\section{Figures}




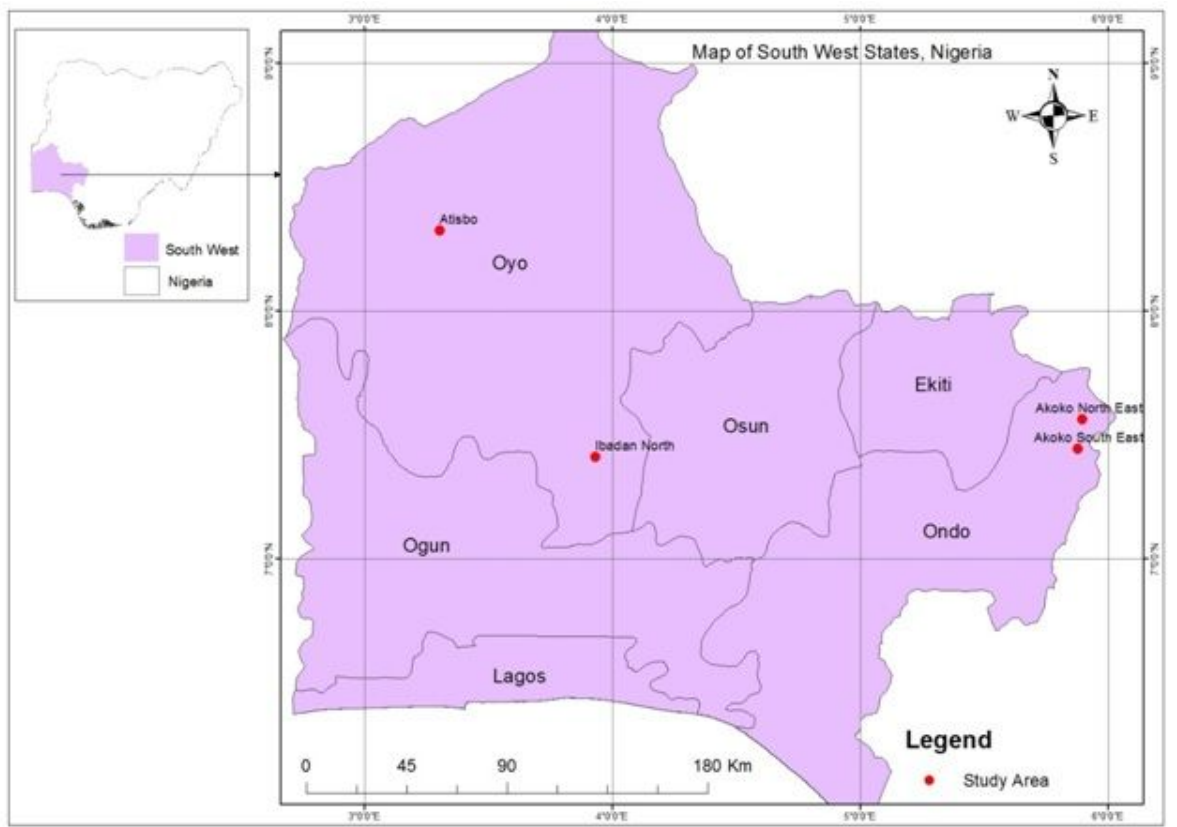

Figure 1

Map of southwestern Nigeria showing the study area

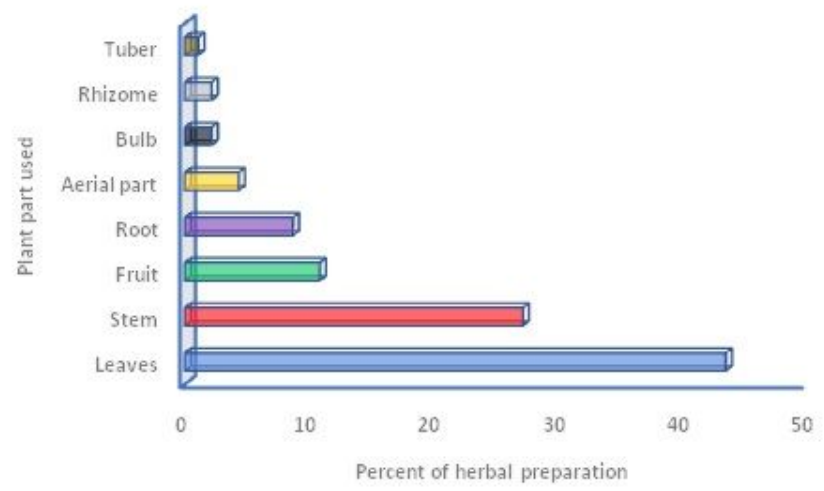

Figure 2

Medicinal plant parts used in the preparation of various remedies for the treatment of diabetes and its co-morbidities in four local government areas in southwestern Nigeria.

\section{Supplementary Files}

This is a list of supplementary files associated with this preprint. Click to download.

- HerbalrecipesEthnobiology.docx

- HerbalrecipesEthnobiology.docx 